\title{
Shared Autonomy for an Interactive Al System
}

\author{
Sharon Zhou, Tong Mu, Karan Goel, Michael Bernstein, Emma Brunskill \\ Stanford University, 353 Serra Mall, Stanford, CA \\ \{sharonz, tongm, kgoel, msb, ebrun\}@cs.stanford.edu
}

\begin{abstract}
Across many domains, interactive systems either make decisions for us autonomously or yield decision-making authority to us and play a supporting role. However, many settings, such as those in education or the workplace, benefit from sharing this autonomy between the user and the system, and thus from a system that adapts to them over time. In this paper, we pursue two primary research questions: (1) How do we design interfaces to share autonomy between the user and the system? (2) How does shared autonomy alter a user's perception of a system? We present SharedKeys, an interactive shared autonomy system for piano instruction that plays different video segments of a piece for students to emulate and practice. Underlying our approach is a mixedobservability Markov decision process that estimates a user's desired autonomy level based on her performance and attentiveness. Pilot studies revealed that students sharing autonomy with the system learned more quickly and perceived the system as more intelligent.
\end{abstract}

\section{INTRODUCTION}

When we make decisions, interactive systems can help us curate suggestions to select; if a decision feels overwhelming, systems can offer us relief from decision paralysis by recommending only one option. Depending on the task and individual, this level of desired autonomy differs, a trade-off between more guidance (fewer options) and more agency (more options). For example, people learn differently on online systems: some enroll on online learning platforms and choose set curricula to follow in lower autonomy; others compile their own curricula by amassing material for self-study in higher autonomy.

Foundational work in psychology has spurred debate over two opposing phenomena [19, 22]: giving people the autonomy to make decisions across several options, on the one hand, significantly improves their motivation, attentiveness, and performance [20,5], and on the other hand, overwhelms them, diverts their attention, and results in significant decline in performance [21, 25]. Literature that seeks to reconcile them suggests that even within the same task, the level of desired autonomy dif-

Permission to make digital or hard copies of part or all of this work for personal or classroom use is granted without fee provided that copies are not made or distributed for profit or commercial advantage and that copies bear this notice and the full citation on the first page. Copyrights for third-party components of this work must be honored. For all other uses, contact the owner/author(s).

UIST '18 Adjunct October 14-17, 2018, Berlin, Germany

(C) 2018 Copyright held by the owner/author(s).

ACM ISBN 978-1-4503-5949-8/18/10.

DOI: https://doi.org/10.1145/3266037.3266088

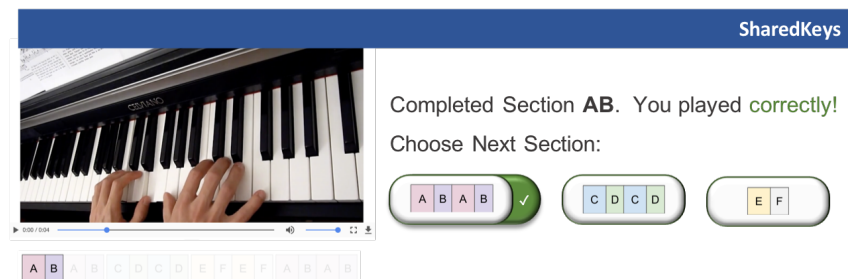

Figure 1: The SharedKeys interface, as a student has correctly completed the video segment (section) AB. The system then gives the student the autonomy to choose which section to practice next: ABAB, CDCD, EF. Had the system not given the student autonomy, section ABAB would have been automatically presented next, but is offered as a suggestion here $(\checkmark)$.

fers between individuals [13] and is contingent on the context in which the person exercises that autonomy [17]. Desired autonomy is difficult to capture, because it is difficult for a system to observe directly and it can be challenging for people to self-assess during a task [17, 13], presenting a compelling design challenge.

Enabling shared autonomy between people and systems, we present SharedKeys, an interactive system for people to learn how to play a piano piece by practicing from different video segments of an expert pianist. Learning music is representative of shared autonomy trade-offs in interactive systems: students desire at times lower autonomy, at other times higher autonomy. We model this shared autonomy using a mixed-observability Markov decision process that maintains a belief over the student's level of desired autonomy using a probability density function $[2,10,18]$. As the system receives feedback on a student's performance and attentiveness over time, this belief is updated to inform the system of the level of autonomy to provide next: to advance to the next recommended video segment, if the system senses that the student prefers lower autonomy; or else to give the student the autonomy to choose the next segment.

\section{RELATED WORK}

Prior work in psychology has demonstrated that student autonomy can either improve $[7,4,5,11,3,12]$ or harm $[21,24,9,25]$ their performance and attentiveness. In fact, students enrolled in the same course demonstrate fluctuating levels of desired autonomy [17] and may need to develop the capacity to operate autonomously before given choices [13]. Previous research also posited value in adapting the level of autonomy as the student learns [1]

Mixed-initiative interfaces infer a user's goals and adapt the interface to either direct manipulation or automation to achieve the user's goals [8]. Adaptive systems also 
feature in cooperative human-robot interactions and employ the concept of shared autonomy $[10,14,15]$ to infer a person's goals [10] or adaptability to the robot [14]. These works leverage mixed-observability Markov decision processes (MOMDPs) [16], a model used in sequential decision-making problems under uncertainty.

Recent HCI research on piano instruction spans haptic feedback [23], a brain-computer interface [26], and memorization exercises [6]. SharedKeys operates an interactive system that adapts to a student's desired autonomy.

\section{SHAREDKEYS}

In this paper we investigate laying shared autonomy on top of an AI system to enable users to learn how to play the first four measures of a Bach piano piece as quickly as possible. The system uses video segments of an expert pianist's performance to teach the music. At each stage of learning, a separate AI system proposes a default music segment for the student to practice next. SharedKeys builds on this system to support user autonomy: SharedKeys has a hierarchical policy, choosing first to give (a) the system or (b) the user autonomy; then (a) following the default curriculum if the system decides or (b) giving the user segment choices if the user decides. To make this decision, SharedKeys maintains both an estimate of the user's desired autonomy, and what is expected to best help her learn quickly given that estimate. The system can interact with each user differently over time, giving or not giving autonomy based on individual performance and attentiveness over time (Figure 2).

Formal Model. More precisely, we formalize shared autonomy using a MOMDP whose latent state space is the learner's desired autonomy (true or false), observable state variables (performance and attentiveness), and action space (giving autonomy and not giving autonomy). In this first iteration of the system, we measure state variables using binary values. We model the transitions between these variables over time in a dynamic Bayesian network (Figure 3) and set approximate transition values. We run the model through a POMDP solver ${ }^{1}$ to produce a decision tree that determines, in real time, the action that the system should take next, given the observed performance and attentiveness (Figure 2 shows sample trajectories in the decision tree). The SharedKeys policy is optimized to maximize our observations of attentiveness, which we hypothesize will move the student along the curriculum more quickly.

\section{PILOT STUDIES}

Initial pilots of SharedKeys compared the system against a system without shared autonomy. We recruited five participants, all of whom had limited piano experience. Two were in the shared autonomy condition. A posttest survey asked about their reactions to the system's choices, their relationship to the system, their perception of system, and if they would continue learning piano. In the shared autonomy condition, we additionally asked

\footnotetext{
${ }^{1}$ https://github.com/JuliaPOMDP/POMDPs.jl
}

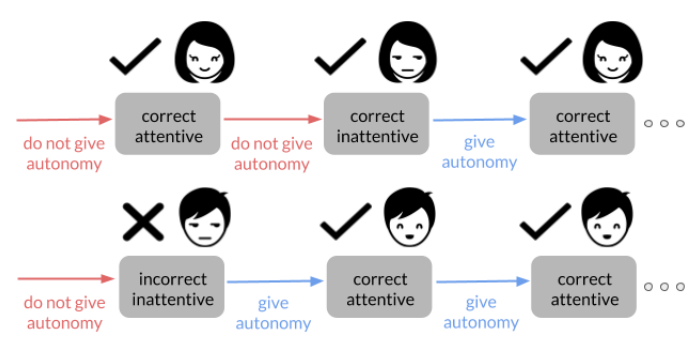

Figure 2: Sample sequences of the system's actions (to give autonomy or to not give autonomy) for different students, based on their performance and attentiveness over time.

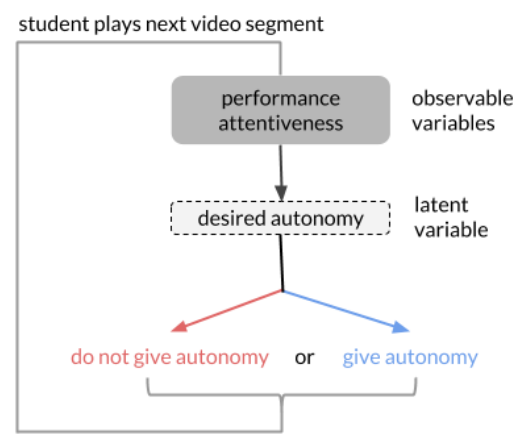

Figure 3: The system observes the user's performance and attentiveness on the first video segment to develop a belief over her desired autonomy. Using this belief, it chooses to give or not give autonomy. The user plays the next segment, chosen either by the system or herself. This process continues until she completes the piece.

whether they enjoyed exercising autonomy and how that impacted their relationship with the system.

Shared autonomy participants all completed the task successfully within an hour, while one participant without shared autonomy did not complete the task and quit. We observed shared autonomy to increase participants' self-reported trust in the system, and led them to believe that the system was more intelligent and in fact helpful. Participants with shared autonomy anthropomorphized the system, which prompted them to reflect on their own performance. Overall, participants were divided in both conditions to learning piano further.

\section{CONCLUSION}

While we developed a system for education, our MOMDP model generalizes to other systems for shared autonomy to help a user maximize performance and attentiveness. We plan to run a full experiment across many users to test our hypothesis: shared autonomy enables students to learn more quickly than randomly or always giving autonomy in this domain.

\section{ACKNOWLEDGMENTS}

We thank the Schmidt Foundation, Stanford AI lab, the National Science Foundation Career Grant \& GRFP, Stanford Graduate Fellowship, and the National Physical Science Consortium Fellowship for their support. 


\section{REFERENCES}

1. C. Ames and J. Archer. Achievement goals in the classroom: Students' learning strategies and motivation processes. Journal of educational psychology, 80(3):260, 1988.

2. B. Brunner, G. Hirzinger, K. Landzettel, and J. Heindl. Multisensory shared autonomy and tele-sensor-programming-key issues in the space robot technology experiment rotex. In Intelligent Robots and Systems' 93, IROS'93. Proceedings of the 1993 IEEE/RSJ International Conference on, volume 3, pages 2123-2139. IEEE, 1993.

3. D. I. Cordova and M. R. Lepper. Intrinsic motivation and the process of learning: Beneficial effects of contextualization, personalization, and choice. Journal of educational psychology, 88(4):715, 1996.

4. E. Deci and R. M. Ryan. Intrinsic motivation and self-determination in human behavior. Springer Science \& Business Media, 1985.

5. E. L. Deci and R. M. Ryan. The" what" and" why" of goal pursuits: Human needs and the self-determination of behavior. Psychological inquiry, 11(4):227-268, 2000.

6. E. Ghomi, S. Huot, O. Bau, M. Beaudouin-Lafon, and W. E. Mackay. Arpège: learning multitouch chord gestures vocabularies. In Proceedings of the 2013 ACM international conference on Interactive tabletops and surfaces, pages 209-218. ACM, 2013.

7. H. Holec, C. of Europe. Council for Cultural Co-operation, and C. of Europe. Autonomy and foreign language learning. Council of Europe modern languages project. Council of Europe, 1981.

8. E. Horvitz. Principles of mixed-initiative user interfaces. In Proceedings of the SIGCHI conference on Human Factors in Computing Systems, pages 159-166. ACM, 1999.

9. S. S. Iyengar and M. R. Lepper. Rethinking the value of choice: a cultural perspective on intrinsic motivation. Journal of personality and social psychology, 76(3):349, 1999.

10. S. Javdani, S. S. Srinivasa, and J. A. Bagnell. Shared autonomy via hindsight optimization. arXiv preprint arXiv:1503.07619, 2015.

11. R. A. Kusurkar, G. Croiset, and O. T. J. Ten Cate. Twelve tips to stimulate intrinsic motivation in students through autonomy-supportive classroom teaching derived from self-determination theory. Medical teacher, 33(12):978-982, 2011.

12. I. Lee. Supporting greater autonomy in language learning. ELT Journal, 1998.

13. B. McCombs. Developing responsible and autonomous learners: A key to motivating students. http://www . apa.org/education/k12/learners . aspx.
14. S. Nikolaidis, J. Forlizzi, D. Hsu, J. Shah, and S. Srinivasa. Mathematical models of adaptation in human-robot collaboration. arXiv preprint arXiv:1707.02586, 2017.

15. S. Nikolaidis, Y. X. Zhu, D. Hsu, and S. Srinivasa. Human-robot mutual adaptation in shared autonomy. In Proceedings of the 2017 ACM/IEEE International Conference on Human-Robot Interaction, pages 294-302. ACM, 2017.

16. S. C. Ong, S. W. Png, D. Hsu, and W. S. Lee. Planning under uncertainty for robotic tasks with mixed observability. The International Journal of Robotics Research, 29(8):1053-1068, 2010.

17. S. Raidal and S. Volet. Preclinical students' predispositions towards social forms of instruction and self-directed learning: a challenge for the development of autonomous and collaborative learners. Higher Education, 57(5):577-596, 2009.

18. S. Reddy, S. Levine, and A. Dragan. Shared autonomy via deep reinforcement learning. arXiv preprint arXiv:1802.01744, 2018.

19. R. M. Ryan and E. L. Deci. Self-determination theory and the facilitation of intrinsic motivation, social development, and well-being. American psychologist, 55(1):68, 2000.

20. R. M. Ryan and E. L. Deci. Self-regulation and the problem of human autonomy: does psychology need choice, self-determination, and will? Journal of personality, 74(6):1557-1586, 2006.

21. B. Schwartz. The paradox of choice: Why more is less, volume 6. HarperCollins New York, 2004.

22. B. Schwartz and A. Ward. Doing better but feeling worse: The paradox of choice. Positive psychology in practice, pages 86-104, 2004.

23. C. E. Seim, D. Quigley, and T. E. Starner. Passive haptic learning of typing skills facilitated by wearable computers. In CHI'14 Extended Abstracts on Human Factors in Computing Systems, pages 2203-2208. ACM, 2014.

24. B. Shiv, Z. Carmon, and D. Ariely. Placebo effects of marketing actions: Consumers may get what they pay for. Journal of marketing Research, 42(4):383-393, 2005.

25. A. Toffler. Future shock. Amereon Ltd., New York, 1970.

26. B. F. Yuksel, K. B. Oleson, L. Harrison, E. M. Peck, D. Afergan, R. Chang, and R. J. Jacob. Learn piano with bach: An adaptive learning interface that adjusts task difficulty based on brain state. In Proceedings of the 2016 chi conference on human factors in computing systems, pages 5372-5384. ACM, 2016. 